\title{
Specificity and overlap in gene segment-defined antibody repertoires Ramy A Arnaout*1,2,3
}

Address: ${ }^{1}$ Department of Pathology, Brigham and Women's Hospital, Boston, MA 02115 USA, ${ }^{2}$ The Broad Institute, Cambridge, MA 02141 USA and ${ }^{3}$ Program for Evolutionary Dynamics, Harvard University, Cambridge, MA 02138 USA

Email: Ramy A Arnaout* - rarnaout@partners.org

* Corresponding author

Published: 28 October 2005

BMC Genomics 2005, 6: 148 doi:|0.|| 86/|47|-2|64-6-|48
Received: 15 April 2005

Accepted: 28 October 2005

This article is available from: http://www.biomedcentral.com//47/-2/64/6/I48

(C) 2005 Arnaout; licensee BioMed Central Ltd.

This is an Open Access article distributed under the terms of the Creative Commons Attribution License (http://creativecommons.org/licenses/by/2.0), which permits unrestricted use, distribution, and reproduction in any medium, provided the original work is properly cited.

\begin{abstract}
Background: To date several studies have sought to catalog the full suite of antibodies that humans naturally produce against single antigens or other specificities (repertoire). Here we analyze the properties of all sequenced repertoires in order to better understand the specificity of antibody responses. Specifically, we ask whether the large-scale sequencing of antibody repertoires might provide a diagnostic tool for detecting antigen exposure. We do this by examining the overlap in $\mathrm{V}_{\mathrm{H}^{-}}$, D-, and $\mathrm{J}_{\mathrm{H}^{-}}$segment usage among sequenced repertoires.
\end{abstract}

Results: We find that repertoire overlap in $\mathrm{V}_{H^{-}}$, $\mathrm{D}$-, and $\mathrm{J}_{H^{-}}$-segment use is least for $\mathrm{V}_{H}$ segments and greatest for $J_{H}$ segments, consistent with there being more $\mathrm{V}_{H}$ than $J_{H}$ segments in the human genome. We find that for any two antigens chosen at random, chances are 90 percent that their repertoires' $V_{H}$ segments will overlap by less than half, and 98 percent that their $V D J_{H}$ combinations will overlap by $\leq 10$ percent. We ran computer simulations to test whether enrichment for specific VDJ ${ }_{H}$ combinations could be detected in "antigen-exposed" populations, and found that enrichment is detectable with moderate-to-high sensitivity and high specificity, even when some VDJ $J_{H}$ combinations are not represented at all in some test sets.

Conclusion: Thus, as large-scale sequencing becomes cost-effective for clinical testing, we suggest that sequencing an individual's expressed antibody repertoire has the potential to become a useful diagnostic modality.

\section{Background}

The antigen-binding variable regions of antibody molecules draw combinatorially from a set of somatically encoded V, D, and J gene segments [1]. Mathematically, this strategy allows for $\sim 6,000$ possible heavy chain (subscript $_{H}$ ) and $\sim 300$ possible light chain ( subscript $_{L}$ ) V(D)J combinations, for a total of $\sim 1.8$ million possible heavyand-light chain pairings $[2,3]$.
Much work in immunology and structural biology has gone into studying how antibody sequence and structure affect antigen specificity [1]. In each antibody, contact with the antigen is made by six short regions, three on each heavy and light chain. These are known as the complementarity-determining regions (CDRs). CDR1 and CDR2 lie entirely within the $\mathrm{V}$ segment, while CDR3 spans the D segment and flanking parts of $\mathrm{V}$ and $\mathrm{J}$ (in heavy chain; in light chain, which lacks a D segment, CDR3 spans the V-J junction). In general, heavy chain 
contributes more than light chain to antigen binding and specificity, and CDR3 contributes more than CDR1 and CDR2 [4]. Hence heavy chain VDJ $\left(\mathrm{VDJ}_{H}\right)$ segment usage is a major determinant of antigen specificity.

There are other determinants. The part of an antigen that an antibody binds is called an epitope; the part of an antibody that an epitope binds is called a paratope. Single antigens may have multiple epitopes, and single antibodies may have multiple paratopes $[5,6]$. Moreover, nontemplated nucleotide insertions and deletions at gene segment junctions, together with CDR hypermutation, expand antibody diversity and antigen binding possibilities far beyond what is available through $\mathrm{V}(\mathrm{D}) \mathrm{J}$ combinatorics alone [1]. Hence V(D)J segment choice and sequence-level modification provide coarse- and fine-tuning, respectively, for antigen specificity, but different $\mathrm{V}(\mathrm{D}) \mathrm{J}$ and sequence combinations may well bind the same antigen.

These considerations and substantial experimental data (summarized in [4]) argue against a strict one-to-one relationship between antibody sequence and antigen specificity. However, they do suggest the possibility that antigens may have signature antibody repertoires. Here a repertoire is defined as a set of antibodies, defined by gene segment usage, that is produced in a population of people against a given specificity. A specificity comprises a single epitope, a set of epitopes on a single antigen, or a set of antigens.

To date several studies have addressed this idea in particular instances by sequencing antibodies specific for particular antigens. In one such study, circulating B cells from seven infants vaccinated against Hemophilus influenzae type b (Hib) were affinity enriched aganst Hib capsular polysaccharide (PS); rearranged V(D)J heavy and light chain gene libraries were then constructed and screened for Hib PS-specific antibodies [7]. The antibodies recovered all used the same $\mathrm{V}_{H}$ segment $\left(\mathrm{V}_{H} 3-23\right)$ and only two $\mathrm{J}_{H}$ and two $\mathrm{V}_{L}$ and $\mathrm{J}_{L}$ segments, consistent with previous studies $[8,9]$. This is consistent with the pattern seen in natural antibody populations, allowing consideration of data from this in vitro "scrambling" approach.

Repertoires against other antigens have also been shown to have restricted segment usage, although the degree and pattern of restriction vary. For example, using a technique similar to that described for Hib PS, the repertoire against Streptococcus pneumoniae serotype 23F PS was found to be dominated by four $\mathrm{V}_{H}$ segments, which account for 90 percent of the repertoire's observed $\mathrm{V}_{H}$ diversity; four $\mathrm{J}_{H}$ segments (93\% of $\mathrm{J}_{H}$ diversity); and two $\mathrm{V}_{L}$-kappa segments (93\%) [10]. For comparison, the repertoire against S. pneumoniae serotype 6B PS was found to be dominated by three $\mathrm{V}_{H}$ segments (93\%) and three $\mathrm{J}_{H}$ segments (98\%), but was found to lack strong $\mathrm{V}_{L}$-kappa restriction (90\% in six segments) [11]. Association patterns among segments and chains were also found to vary.

In all, repertoires for over a dozen antigens have been studied individually, with various aims and to various extents, mainly through enrichment and cloning or through screening of phage-display libraries [7,10-14]. The aim of the present study is to analyze these repertoires as a group in order to better understand the specificity of antibody responses. The practical goal is to explore the possibility that in the future, large-scale sequencing of antibodies in an individual may be used as a fingerprint, or "pan-scan," of that person's antigen exposure.

\section{Results}

We analyzed $\mathrm{VDJ}_{H}$ segment usage for the 16 best-represented natural human repertoires in the IMGT database (see Methods). These comprised 292 antibody sequences (mean, 18 sequences per repertoire; range, 8-41). Six repertoires were directed against infectious agents, while 10 were directed against autoimmune agents (Table 1 and Additional File 1).

\section{Gene segment usage patterns}

Genome-level diversity was well represented among the repertoires as a group. All but one $\left(\mathrm{V}_{H} 7\right)$ of the $\mathrm{V}_{H}$ and $\mathrm{D}$ gene segment families were represented, and the majority of individual $\mathrm{V}_{H}(78 \%), \mathrm{D}(91 \%)$, and $\mathrm{J}_{H}(100 \%)$ gene segments appeared in at least one sequence. $\mathrm{V}_{H}$ and $\mathrm{D}$ gene families were represented about as often as in a previous study of healthy individuals [15] ( $p=0.01$ and 0.13 , $R^{2}=0.78$ and 0.96 for $\mathrm{V}_{H}$ and $\mathrm{D}$ families, respectively), as were individual $\mathrm{J}_{H}$ gene segments $\left(p=0.004, R^{2}=0.94\right)$. However, individual $\mathrm{V}_{H}$ gene segments were used more variably $\left(p=0.90, R^{2}=0.25\right)$.

These observations are consistent with there being more than one $\mathrm{VDJ}_{H}$ combination used in antibodies with a given specificity (see below). They also suggest either that our set of repertoires is a good representation of at least the kinds [16] of antigen or antigen patterns encountered naturally, or conversely that $\mathrm{B}$ cell populations of the healthy individuals sampled in the previous study [15] comprise clones expanded against specificities similar to the ones included in our present analysis. These possibilities are not mutually exclusive.

Figure 1 shows $\mathrm{V}_{H}, \mathrm{D}$, and $\mathrm{J}_{H}$ segment usage and $\mathrm{VDJ}_{H}$ combination usage patterns for the repertoires of representative specificities. Some repertoires were peaked and narrow, suggesting few epitopes or immunodominance among the epitopes in their specificities, or little diversity among individuals for these specificities ("public" or "semi-public" repertoires; see Discussion). Other 
Table I: Repertoire composition

\begin{tabular}{|c|c|c|c|c|c|}
\hline specificity & sequences & $V_{H}$ genes & D genes & $J_{H}$ genes & $\mathrm{VDJ}_{H}$ combos \\
\hline E. histolytica & 9 & 7 & 5 & 2 & 7 \\
\hline $\mathrm{HBsAg}(\mathrm{HBV})$ & 12 & 9 & 8 & 3 & 11 \\
\hline PS (S. pneumo 23F) & 23 & 7 & 10 & 4 & 15 \\
\hline gpI 20 (HIV) & 26 & 10 & 16 & 6 & 24 \\
\hline PS (S. pneumo 6B) & 41 & 5 & 10 & 3 & 11 \\
\hline dsDNA (human) & 8 & 7 & 6 & 4 & 8 \\
\hline MAG (human) & 9 & 7 & 7 & 4 & 9 \\
\hline PL (human) & 9 & 8 & 8 & 4 & 9 \\
\hline Fab (human) & 11 & 9 & 7 & 3 & 10 \\
\hline factor VIII (human) & 19 & 3 & 5 & 3 & 6 \\
\hline cardiolipin (human) & 12 & 7 & 7 & 3 & 10 \\
\hline gpllb/Illa (human) & 14 & 12 & 10 & 3 & 14 \\
\hline myosin (human) & 14 & 12 & 10 & 5 & 14 \\
\hline RhD (human) & 22 & 9 & 14 & 5 & 20 \\
\hline DNA (human) & 22 & 12 & 13 & 4 & 20 \\
\hline TPO (human) & 41 & 6 & 11 & 4 & 16 \\
\hline total & 292 & 36 & 21 & 6 & 192 \\
\hline
\end{tabular}

Number of sequences, gene segments, and gene segment combinations in the repertoires of all specificities in the data set. Specificities associated with infectious agents listed first; species names within parentheses (where necessary). Abbreviations: E. histolytica, Entameba histolytica; HBV, hepatitis B virus; PS, polysaccharide; S. pneumo, Streptococcus pneumoniae; ds, double-stranded; MAG, myelin-associated glycoprotein; PL, phospholipid; TPO, thyroid peroxidase.

repertoires were flat and broad, suggesting many epitopes or codominance among the epitopes in their specificities, or greater diversity among individuals for these specificities. Details of $\mathrm{V}_{H}, \mathrm{D}$, and $\mathrm{J}_{H}$ segment usage for particular specificities have been discussed elsewhere (see references for specific sequences in IMGT, Table 1, and Additional File 1).

The data did not allow conclusive generalization about whether or not, for a given repertoire, $\mathrm{V}_{H^{\prime}} \mathrm{D}$, and $\mathrm{J}_{H}$ segments are combined randomly or with some bias. This is because the number of antibodies sequenced in a given repertoire was small (8-41 sequences) relative to the number of $\mathrm{VDJ}_{H}$ combinations that could in principle be constructed from the $\mathrm{V}_{H^{\prime}} \mathrm{D}$, and $\mathrm{J}_{H}$ segments that appeared in that repertoire ( $50-1,000$ possibilities).

For only one of the 16 repertoires - the repertoire for thyroid peroxidase - was there a tight, statistically significant correlation between the observed frequencies of $\mathrm{VDJ}_{H}$ combinations and the frequencies that would be expected if segments were combined at random $\left(p<0.01 ; R^{2}=\right.$ 0.85 ). The repertoire for $S$. pneumoniae strain $6 \mathrm{~B}$ polysaccharide also showed a tight correlation, but this correlation fell short of statistical significance $\left(p=0.10 ; R^{2}=\right.$ $0.95)$. No tight, statistically significant correlation was observed for any other repertoire. These findings are consistent with the conclusion that $\mathrm{V}_{H}, \mathrm{D}$, and $\mathrm{J}_{H}$ segments are not joined at random in at least 14 of these 16 repertoires, but more sequencing is needed to settle this issue.

\section{Overlap in gene segment usage}

From a practical perspective, for repertoires to serve as signatures for particular specificities, the overlap in gene segments or in V(D)J combinations among different repertoires must be low. To estimate this overlap quantitatively, we calculated the percent overlap between each pair of specificities in the data set (Fig. 2).

We found that for any two specificities picked at random from our set, the probability was 90 percent that their repertoires' $\mathrm{V}_{H}$ gene segment usage overlapped by half or less (Fig. $2 \mathrm{a}$, red tones). Adding $\mathrm{D}$ and $\mathrm{J}_{H}$ segment information decreased the overlap markedly: of the 240 pairwise comparisons between different specificities in our data set, only four $(1.7 \%)$ showed more than 10 percent overlap: between dsDNA and RhD (12\%), thyroid peroxidase (TPO) and factor VIII (16\%), TPO and phospholipid (11\%), and phospholipid and integrin gpIIb/IIIa (11\%) all autoimmune specificities. Although not random in segment usage, autoimmune antibodies may share common features that result from impaired negative selection. Overall, for any two specificities chosen at random, the probability was 98.3 percent that their repertoires' $\mathrm{VDJ}_{H}$ combinations overlapped by 10 percent or less (Fig. 2b).

Given the large number of possible $\operatorname{VDJ}_{H}$ combinations $(\sim 6,000)$ and the relatively small size of the data set $(292$ sequences), it is reasonable to ask whether or not such a small amount of overlap is likely to occur by chance. Probability calculations show that it is not. The two most 
factor VIII
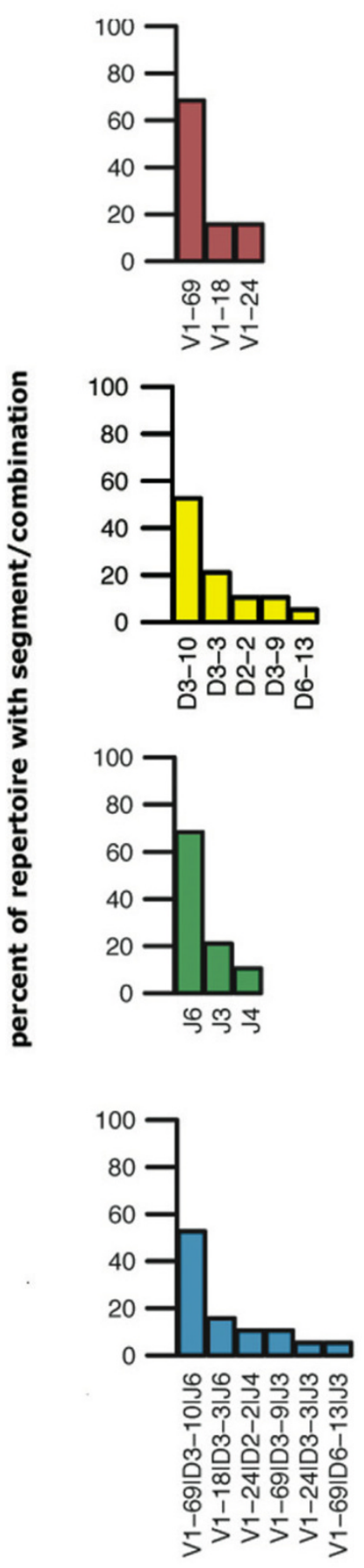

S. pneumoniae 6B PS
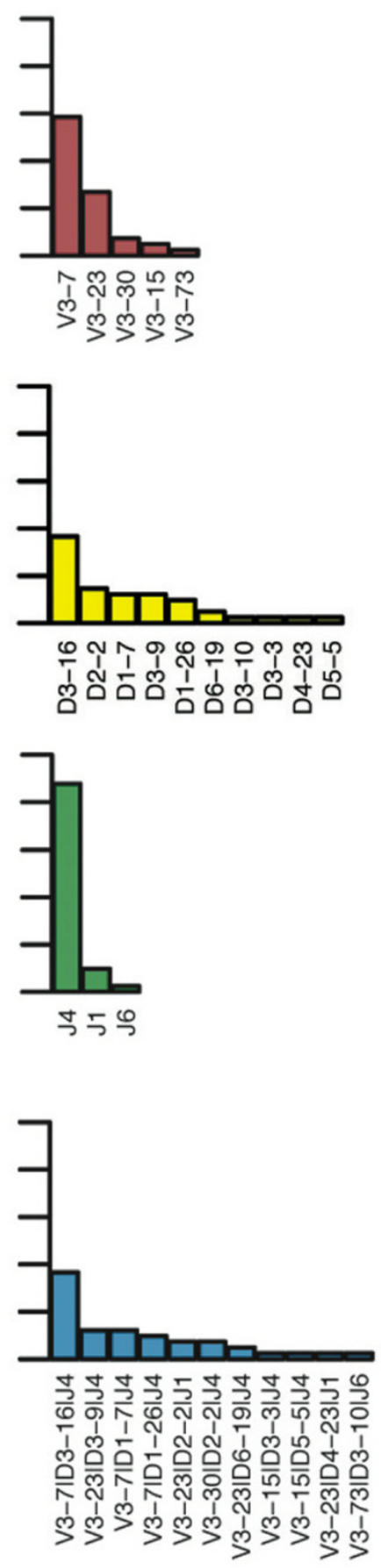

\section{S. pneumoniae 23F PS}
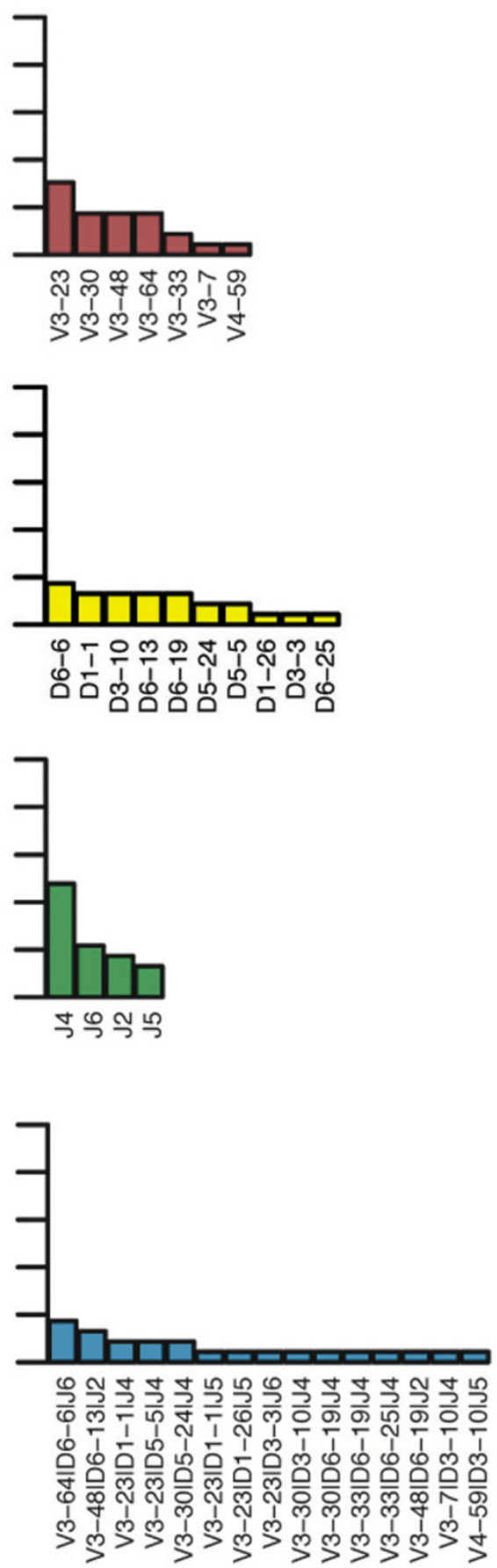

\section{gene segment/combination used}

\section{Figure I}

Gene segment use for representative repertoires. Repertoires for three specificities are shown: human coagulation factor VIII, Streptococcus pneumoniae serotype 6B capsular polysaccharide (PS), and S. pneumoniae ser. 23F PS. Each histogram shows the frequency distribution of $V_{H}$ gene segments, $D$ segments, $J_{H}$ segments, and $V D J_{H}$ combinations. More peaked distributions indicate that the repertoire is $\mathrm{V}_{H}, \mathrm{D}, \mathrm{J}_{H}$, or $\mathrm{VD} \mathrm{J}_{H}$ restricted. For example, the $\mathrm{S}$. pneumoniae ser. $6 \mathrm{~B}$ repertoire is $80 \%$ restricted to $\mathrm{J}_{H}$ gene segment $\mathrm{J}_{H} 4$. 


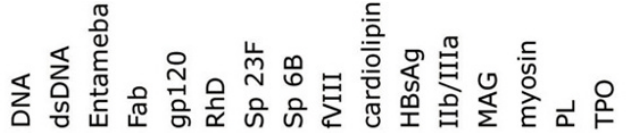

a

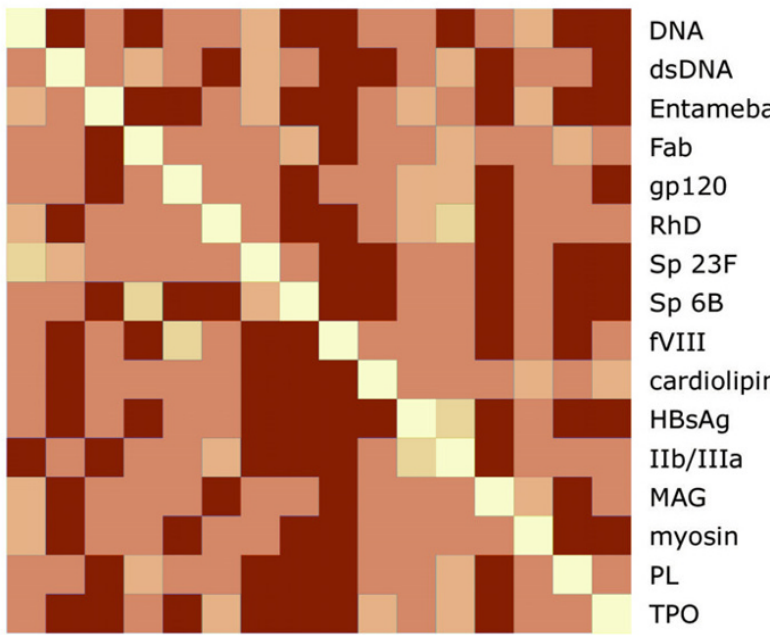

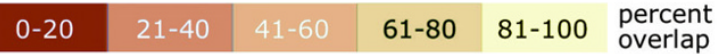

b

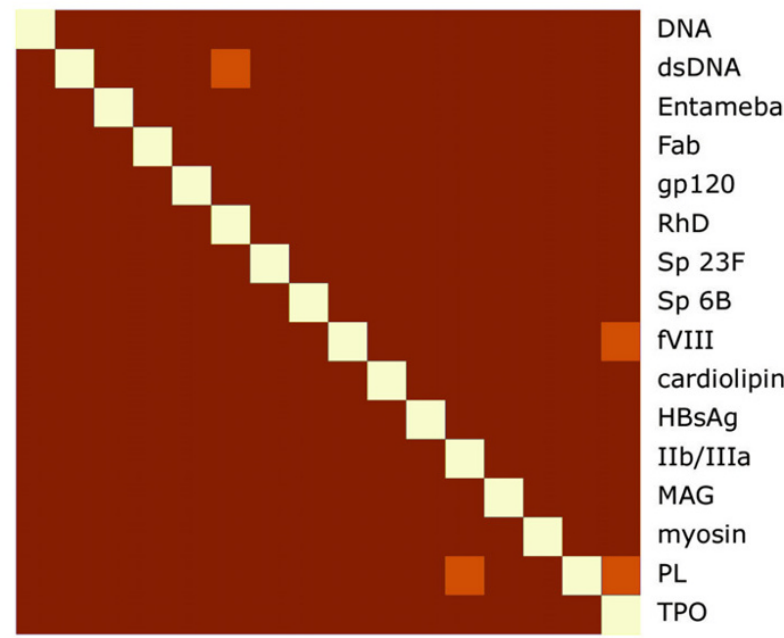

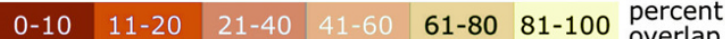

Figure 2

Overlap in segment use among repertoires. Overlap in (a) $\mathrm{V}_{H}$ gene segment and (b) $V D J_{H}$ use among all repertoires. The percent overlap is grayscale-coded according to the key below each plot. In (b), the range at the lower end of the scale is expanded in order to show the four pairs with $11-20$ percent overlap (see text). Abbreviations: ds-DNA, doublestranded DNA; gp I20, HIV-I gp I20; Sp, Streptococcus pneumoniae serotype; fVIII, clotting factor VIII; HBsAg, HBV surface antigen; Ilb/llla, glycoprotein Ilb/Illa; MAG, myelinassociated glycoprotein; PL, phospholipid; TPO, thyroid peroxidase. Species of origin are as in Table I. common human haplotypes allow a maximum of 5,244 and 6,348 possible functional $\mathrm{VDJ}_{H}$ combinations, respectively; the probability that the small amount of overlap observed in our data should arise by chance is $p=$ $0.004(0.4 \%)$ and $0.011(1.1 \%)$ for these two haplotypes, respectively (see Methods). Note that nonrandom association among $\mathrm{V}_{H^{\prime}}, \mathrm{D}$, and $\mathrm{J}_{H}$ segments means that only a fraction of these 5,244 or 6,348 possible combinations are actually observed. The smaller the number of combinations, the higher the probability that repertoires will overlap by chance. Hence the small amount of overlap observed in the data is even less likely to be the result of chance than these calculations suggest. The probabilities are therefore upper limits.

If the specificities analyzed in this study are indeed representative of the specificities to which human beings are exposed (see above), this finding suggests that $\mathrm{VDJ}_{H^{-}}$ defined sequences may be able to distinguish dependably among a wide variety of specificities.

\section{Simulating detection}

For repertoires to be of practical use, it must be possible to detect when certain $\mathrm{VDJ}_{H}$ combinations are present at a higher-than-background frequency. This may indicate, for example, prior or ongoing exposure to an infectious agent or the presence of a response to a vaccine [17]. Ideally detection should be possible even when this frequency is barely above background - that is, when the signal-tonoise ratio is low.

To test whether enrichment might be detectable, we ran computer simulations for each specificity. These were done briefly as follows (for details, see Methods). For each specificity, we assembled several sets of sequences that were each enriched for sequences of that specificity's repertoire. (The analogy is that each set of sequences corresponds to what might be obtained from a blood sample of an individual known to have a clinical history of that specificity.) The collection of these sets was our "reference collection" for the test (medically, the gold standard). The strategy was to see if test sets could be assigned as exposed or unexposed by comparing their patterns of $\mathrm{VDJ}_{H}$ combinations to the ones from the reference collection. If antibodies in a test set had a similar pattern and prevalence of $\mathrm{VDJ}_{H}$ combinations as those in the reference collection, the test set was assigned as "exposed." If the patterns were dissimilar, the test set was assigned as "unexposed." Assignment was performed with the aid of a computerized algorithm (see Methods).

We tested this approach for each specificity by seeing how well exposed and unexposed sets could be assigned. In clinical infections, B cells specific for an infectious agent rarely exceed 5-10 percent of the total B cell population. 

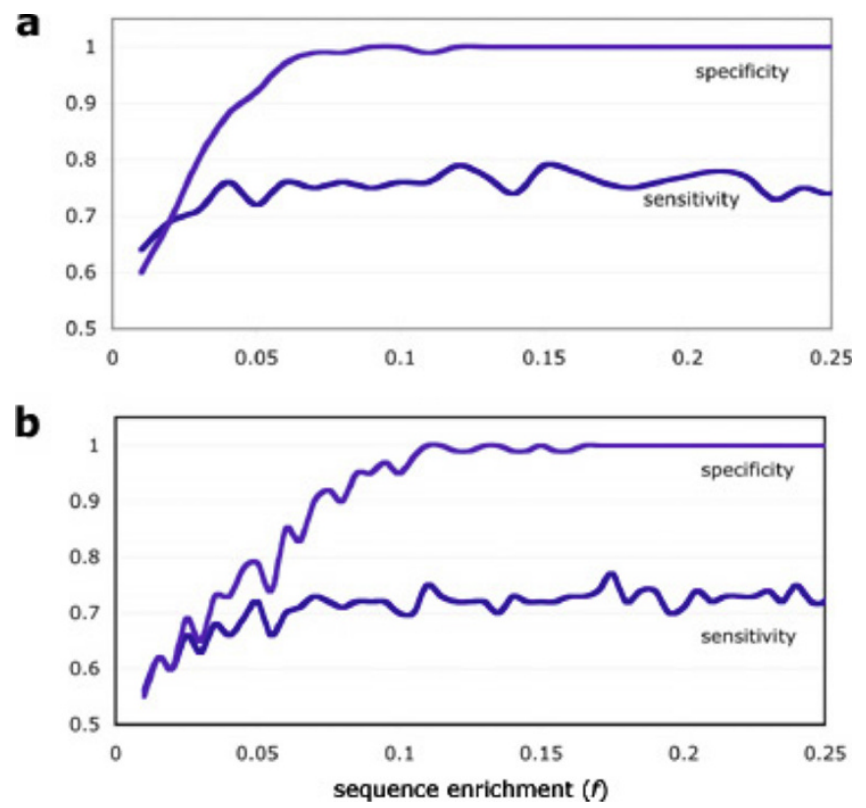

Figure 3

Detection of exposure to representative specificities. Representative plots of sensitivity and specificity for detecting exposure at various levels (f; see Methods for details): (a) Streptococcus pneumoniae serotype 6B and (b) S. pneumoniae ser. 26F. In general sensitivities reached between 0.7 and 0.8 when repertoires were enriched to at least 5 percent $(f=$ 0.05 ), and specificities reached between 0.95 and $\mathrm{I}$.

Therefore, as a conservative test, the sets in the reference collection had only 1-2.5 percent of their $\mathrm{VDJ}_{H}$ combinations purposely drawn from the repertoire for the given specificity. For example, in testing for exposure to HIV gp120, of 1,000 $\mathrm{VDJ}_{H}$ combinations determined for a set in the reference collection, only 10-25 would be guaranteed to be combinations that appeared in the HIV gp120 repertoire; the rest would be from the repertoires of $S$. pneumoniae serotype 6B PS, double-stranded (ds) DNA, and the other 14 specificities. Note that in this approach not all combinations are guaranteed to appear in any one set; however, the more frequently a combination appears in the repertoire - the higher its prevalence - the more likely (and more often) it is to appear in a given set. Also, the larger the reference collection, the more likely that less prevalent combinations will also appear in at least one set.

A training collection for each specificity was assembled comprising 10 exposed and 10 unexposed sets. An additional 50 test sets, whose exposed/unexposed status was known to us but not to the algorithm, were presented for assignment. Performance was measured by sensitivity and specificity (see Methods). Figure 3 shows results for two typical simulations. Sensitivity generally reached between 0.7 and 0.8 when exposure-specific antibodies/sequences were five percent of the total; specificity was higher (most likely due to false negatives in the sensitivity because of the small size of the reference sets). Sensitivity was improved by increasing the size of and enrichment in the sets in the reference collection. (Here the terms "sensitivity" and "specificity" are used in the epidemiological sense; see Methods.)

\section{Discussion}

The majority of modern clinical tests assay for just one analyte at a time [18]. They determine the presence or absence of the analyte, and sometimes its quantity, but provide no information about other analytes. For example, a nucleic acid test for HIV-1 determines whether or not HIV-1 RNA is present in blood, and how much, but provides no information about, for example, the presence of antibodies to CMV. Although such tests are the mainstay of modern medicine, conceptually, they are limited to providing a "20 questions," yes-or-no approach to diagnosis.

The major exception is the standard culture-based method for diagnosing bacterial infections. In this method, the first step is to apply a clinical sample to standard culture media to see what grows [19]. This method is powerful in that it presupposes little about the identity of the bacteria: it can distinguish among many bacteria with a single test, and often reveals the presence of species that were clinically unexpected. Conceptually, this is an open-ended, "what-is-there" approach to diagnosis. It is of general interest in medicine to develop more diagnostic techniques that use this approach.

Antibodies play a crucial role in protective immunity and immunopathology, and also are important in surveillance against cancer [1]. The relationship between antibody gene sequence and epitope specificity is complex, but several studies have shown that certain gene segments and gene segment combinations are used preferentially against specific epitopes, antigens, or sets of antigens what we here call "specificities" [7,10-14]. The identity and frequency of gene segments or combinations define antibody repertoires.

In this paper we have analyzed the growing, albeit limited, data that exists on $\mathrm{VDJ}_{H}$ combination defined repertoires to see whether they might one day provide an open-ended diagnostic for antigens to which a person has been exposed. For statistical confidence, we analyzed only those specificities for which at least eight antibodies have been sequenced and annotated for $\mathrm{V}_{H}, \mathrm{D}$, and $\mathrm{J}_{H}$ gene segment use. A similar amount of systematic data for immunoglobulin light chains and $\mathrm{T}$ cell receptors is still 
unavailable, and so the present analysis was limited to immunoglobulin heavy chains.

Our data set represented nearly every gene segment family, and at frequencies similar to those seen in two healthy individuals in a previous study [15]. One interpretation is that this reflects an intrinsic bias in the frequency with which different $\mathrm{VDJ}_{H}$ combinations are formed or expressed. Another interpretation is that the specificities in our data set are representative of the exposures that shape repertoires in healthy individuals, since certain types of antigens - bacterial polysaccharides, for instance - select for certain canonical structures in antibodies, and segments of the same gene family are more likely to produce similar structures [16]. These two interpretations are not mutually exclusive.

The narrowness or breadth of the repertoires for individual specificities (Fig. 2) could simply reflect the number of epitopes per specificity. For example, the antibodies against factor VIII, which formed a narrow repertoire, are known to have been raised against relatively well defined domains of factor VIII that comprise few epitopes [20], while antibodies against dsDNA, which formed a broad repertoire, were not raised this way [21].

The fact that the same $\mathrm{VDJ}_{H}$ combinations were recovered from multiple individuals in many repertoires (e.g., the $S$. pneumoniae PS repertoires $[10,11])$ suggests that despite genetic differences, different individuals may often use the same or at least overlapping sets of $\mathrm{VDJ}_{H}$ combinations in the antibodies they make against a given epitope. These could be called "public" or "semi-public" combinations [7,3]. Such commonalities might shed light on the evolutionary forces - repeat exposure to particular infectious agents, for example [4] - that may have shaped and maintained germline gene segment diversity. Further sequencing experiments using specificities defined the epitope level would be useful to determine how often and to what epitopes public and semi-public combinations occur. The more frequent public combinations turn out to be, the more narrowly defined specificities can be and remain detectable, and vice versa.

Repertoires' $\mathrm{VDJ}_{H}$ combinations overlapped rarely (Fig. $3 \mathrm{~b})$, and less often than would be predicted by chance ( $p$ $\leq 0.011$ ). Specifically, for any two specificities chosen at random, chances were 98.3 percent that they overlapped by 10 percent or less. This suggests that determining VDJ $_{H}$ usage for a sampling of antibodies can be used to identify exposure to a particular antigen or set of antigens with reasonable specificity.

To further explore this idea, we conducted a set of simulation experiments to see whether individuals could one day be diagnosed as being exposed or not exposed to a given specificity (relative to a normal baseline) by assaying for enrichment of certain $\mathrm{VDJ}_{H}$ combinations. We show that even at modest levels of enrichment, which represents an increased frequency of B cells specific to a certain exposure, and using just 10 reference sets as the "gold standard" for exposure, assignment of unknown sets as either exposed or unexposed was possible with a high degree of sensitivity and specificity. In principle, such a sequence-based method has the advantage of being able to detect patterns of exposure even when the specificity of the antibodies or the identity of the offending agent is completely unknown. This "open-ended" approach is most useful for the early detection of emerging diseases, and will become practicable as improvements in sequencing technology make it possible to use in the clinic [22]. Data on antibody titers and functionality will doubtless add to the utility of this approach.

\section{Conclusion}

In sum, this study is the first to our knowledge that investigates the relationship between antibody specificity and $\mathrm{VDJ}_{H}$ segment usage for a large number of sequenced antibodies. Further sequencing studies should make it possible to refine the conclusions presented here, and also to assess the contribution of light chain in antibodies and of alpha and beta chains in T cell receptors to antigen specificity in human immune responses. Whether or not largescale sequencing will prove useful as a future diagnostic tool will depend on these further studies.

\section{Methods \\ Antibody repertoire data}

The ImMunoGeneTics database (IMGT; http:// imgt.cines.fr/) is a publically available curated online repository of $\sim 88,000$ sequenced immunoglobulin and $\mathrm{T}$ cell receptor genes from a number of species [2]. We extracted all 531 entries that contained recombined human immunoglobulin genes annotated with $\mathrm{V}_{H^{\prime}} \mathrm{D}$, and $\mathrm{J}_{H}$ gene segment use and antigen specificity.

To approximate only natural repertoires, we limited our analysis to sequences isolated from B cells of individuals, and excluded all sequences that had been designed or modified in vitro. Allowed sequences included ones obtained from Epstein-Barr Virus (EBV)-immortalized B cells, through combinatorial cloning or phage-display libraries constructed from B cells of antigen-exposed patients, and from single sequenced B cells. For statistical power we considered only those specificities that had at least eight sequences in IMGT. There were 16 such specificities, comprising a total of 292 individual antibody sequences (mean, 18 sequences per specificity; range, 841). 


\section{Frequency distributions and overlap}

We calculated and tabulated $\mathrm{V}_{H}, \mathrm{D}$, and $\mathrm{J}_{H}$ frequency distributions from all specificities and calculated their pairwise overlap computationally. Because specificities generally differed in the number of unique $\mathrm{VDJ}_{H}$ combinations in their repertoires, overlap was not symmetric: for example, if one specificity's repertoire comprised five different $\mathrm{VDJ}_{H}$ combinations, and another specificity's repertoire had those same five combinations as well as an additional 15, the overlap would be 100 percent in one direction, but only 25 percent in the other.

Student's t-test for two independent samples was used to obtain p-values for calculated vs. observed frequencies of $\mathrm{VDJ}_{H}$ combinations for each repertoire. Heatmap plots were made using $\mathrm{R}$ http://cran.r-project.org/.

Humans most commonly encode 38 functional $V_{H}$ genes, 23 functional $\mathrm{D}$ genes, and 6 functional $\mathrm{J}_{H}$ genes, as well as a number of pseudogenes [2]. These allow for a theoretical maximum of $38 \times 23 \times 6=5,244$ possible $\mathrm{VDJ}_{H} \mathrm{com}$ binations. In addition, many Caucasians contain a partial duplication of the $\mathrm{V}_{H}$ region that results in 46 functional $\mathrm{V}_{H}$ genes [2]; this partial duplication allows for a theoretical maximum of $46 \times 23 \times 6=6,348 \mathrm{VDJ}_{H}$ combinations. For a person with a maximum of 5,244 possible $\mathrm{VDJ}_{H}$ combinations, the probability that two sets of 10 randomly chosen combinations will not overlap at all is approximately $[(5,244-10) / 5,244]^{10}=0.98$, or 98 percent. The probability that a third set of, for example, seven combinations will not overlap at all with either of these two sets is approximately $[(5,244-10) / 5,244]^{10} \times[(5,244$ - $10-10) / 5,244]^{7}=0.96$, or 96 percent. The probability of overlap among any group of sets may be approximated by extending this method.

\section{Simulations}

We built a pattern-detecting computer algorithm for detecting enrichment of antibody sequences that correspond to particular specificities [23] (single-hidden layer, feed-forward neural networks with backpropagation; Brainstem v1.4; http://digforfire.dyndns.org:8847).

For each specificity, the algorithm was trained on a reference collection representing 10 exposed and 10 unexposed sequence sets as follows. Each set constituted a list of the frequency of each of $100 \mathrm{VDJ}_{H}$ combinations drawn equally from all the specificities in the data set. For each specificity, we sampled a fraction $(f)$ of combinations from that specificity's repertoire according to their frequency distribution, allowing resampling. The remainder were sampled from all sequences in the data set, including those of the chosen repertoire, again allowing resampling. This remainder represents a background of noise against which a signal - enrichment of specific sequences - might be detected. $0<f \leq 1$ for exposed sets and $f=0$ for unexposed sets. Hence, a set is "exposed" if it is statistically enriched for sequences a particular specificity, and "unexposed" otherwise. Note that unexposed sets will contain some sequences from the chosen exposed repertoire by chance, just at lower frequency than in exposed sets. Our question was, how well can we assign, or "diagnose," exposure: i.e., how well can we detect enrichment.

The algorithm was used to evaluate test sets, each comprising an additional 25 exposed and 25 unexposed patients. For each specificity, the algorithm was trained at $0.01 \leq f$ $\leq 0.025$ (for the exposed patients) and tested over the range $0.01 \leq f \leq 1$. To quantify the results, we calculated the sensitivity [(true positives)/(true positives + false negatives)] and specificity [(true negatives)/(true negatives + false positives)] of the algorithm for each test set. These are standard metrics for diagnostic tests in the clinical setting [18].

\section{List of abbreviations}

dsDNA, double-stranded DNA; HIV-1, human immunodeficiency virus type 1; Sp, Streptococcus pneumoniae serotype; fVIII, clotting factor VIII; HBsAg, hepatitis B virus surface antigen; IIb/IIIa, glycoprotein IIb/IIIa; MAG, myelin-associated glycoprotein; PL, phospholipid; TPO, thyroid per-oxidase; $\mathrm{CMV}$, cytomegalovirus.

\section{Authors' contributions}

R.A.A. performed all the work presented in this paper.

\section{Additional material}

\section{Additional File 1}

VDJtable.pdf, is a PDF file that contains a table listing VDJ combinations for all specificities analyzed in this paper.

Click here for file

[http://www.biomedcentral.com/content/supplementary/1471-

2164-6-148-S1.pdf]

\section{Acknowledgements}

The author would like to thank Miguel N. Rivera and Eric S. Lander for helpful conversations.

\section{References}

I. Abbas AK, Pober JS, Lichtman AH: Cellular and Molecular Immunology. Saunders 200I.

2. Lefranc MP: IMGT, the international ImMunoGeneTics information system. Novartis Found Symp 2003, 254( I 528-25 I I I 26-36 [http://imgt.cines.fr]. discussion 136-42, 216-22, 250-2

3. Ohlin $M$, Zouali $M$ : The human antibody repertoire to infectious agents: implications for disease pathogenesis. Mol Immunol 2003, 40: I-II.

4. Frank SA: Immunology and Evolution of Infectious Disease. Princeton 2002.

5. James LC, Roversi P, Tawfik DS: Antibody multispecificity mediated by conformational diversity. Science 2003, 299(56 | I): | 362-7. 
6. Richards FF, Konigsberg WH, Rosenstein RW, Varga JM: On the specificity of antibodies. Science 1975, 187(4I72): I30-7.

7. Lucas AH, McLean GR, Reason DC, O'Connor AP, Felton MC Moulton KD: Molecular ontogeny of the human antibody repertoire to the Haemophilus influenzae type $B$ polysaccharide: expression of canonical variable regions and their variants in vaccinated infants. Clin Immunol 2003, I 08(2): I I9-27.

8. Pinchuk GV, Nottenburg C, Milner EC: Predominant V-region gene configurations in the human antibody response to Haemophilus influenzae capsule polysaccharide. Scand J Immunol 1995, 4 I (4):324-30.

9. Adderson EE, Shackelford PG, Quinn A, Wilson PM, Cunningham MW, Insel RA, Carroll WL: Restricted immunoglobulin VH usage and VDJ combinations in the human response to Haemophilus influenzae type b capsular polysaccharide. Nucleotide sequences of monospecific anti-Haemophilus antibodies and polyspecific antibodies cross-reacting with self antigens. J Clin Invest 1993, 9 I(6):2734-43.

10. Zhou J, Lottenbach KR, Barenkamp SJ, Lucas AH, Reason DC: Recurrent variable region gene usage and somatic mutation in the human antibody response to the capsular polysaccharide of Streptococcus pneumoniae type 23F. Infect Immun 2002, 70(8):4083-9I.

II. Zhou J, Lottenbach KR, Barenkamp SJ, Reason DC: Somatic hypermutation and diverse immunoglobulin gene usage in the human antibody response to the capsular polysaccharide of Streptococcus pneumoniae Type 6B. Infect Immun 2004, 72(6):3505-14.

12. Chardes T, Chapal N, Bresson D, Bes C, Giudicelli V, Lefranc MP Peraldi-Roux S: The human anti-thyroid peroxidase autoantibody repertoire in Graves' and Hashimoto's autoimmune thyroid diseases. Immunogenetics 2002, 54(3):14|-57.

13. Sun Y, Park MK, Kim J, Diamond B, Solomon A, Nahm MH: Repertoire of human antibodies against the polysaccharide capsule of Streptococcus pneumoniae serotype 6B. Infect Immun 1999, 67(3): $1172-9$.

14. Uray K, Hudecz F, Fust G, Prohaszka Z: Comparative analysis of linear antibody epitopes on human and mycobacterial 60kDa heat shock proteins using samples of healthy blood donors. Int Immunol 2003, I 5( I 0): 1229-36.

15. Brezinschek HP, Foster SJ, Brezinschek RI, Dorner T, Domiati-Saad R, Lipsky PE: Analysis of the human VH gene repertoire. Differential effects of selection and somatic hypermutation on human peripheral CD5(+)/lgM+ and CD5(-)/IgM+ B cells. J Clin Invest 1997, 99( 10):2488-50 I.

16. Vargas-Madrazo E, Lara-Ochoa F, Almagro JC: Canonical structure repertoire of the antigen-binding site of immunoglobulins suggests strong geometrical restrictions associated to the mechanism of immune recognition. J Mol Biol 1995, 254(3):497-504.

17. Plotkin SA, Orenstein WA, Offit PA, Eds: Vaccines. Saunders 2003.

18. McClatchey KD: Clinical Laboratory Medicine 2nd edition. Lippincott Williams \& Wilkins; 2002.

19. Murray PR, Baron EJ, Jorgensen JH, Pfaller MA, Yolken RH, Eds: Manual of Clinical Microbiology American Society of Microbiology; 2003.

20. van den Brink EN, Bril WS, Turenhout EAM, Zuurveld M, Bovenschen N, Peters M, Yee TT, Mertens K, Lewis DA, Ortel TL, Lollar P, Scandella $D$, Voorberg J: Two classes of germline genes both derived from the $V(H) \mid$ family direct the formation of human antibodies that recognize distinct antigenic sites in the C2 domain of factor VIII. Blood 2002, 99(8):2828-34.

21. Behrendt M, Partridge LJ, Griffiths B, Goodfield M, Snaith M, Lindsey $\mathrm{NJ}$ : The role of somatic mutation in determining the affinity of anti-DNA antibodies. Clin Exp Immunol 2003, I 3 I: 182-9.

22. Shendure J, Mitra RD, Varma C, Church GM: Advanced sequencing technologies: methods and goals. Nat Rev Genet 2004, 5(5):335-44.

23. Fausett LV: Fundamentals of Neural Networks Prentice-Hall; 1994.

\footnotetext{
Publish with Biomed Central and every scientist can read your work free of charge

"BioMed Central will be the most significant development for disseminating the results of biomedical research in our lifetime. " Sir Paul Nurse, Cancer Research UK

Your research papers will be:

- available free of charge to the entire biomedical community

- peer reviewed and published immediately upon acceptance

- cited in PubMed and archived on PubMed Central

- yours - you keep the copyright

Submit your manuscript here:

http://www.biomedcentral.com/info/publishing_adv.asp

BiolMedcentral
} 